CONCLUSIONS: Use of a pharmacogenetic assay was associated with cost-savings in the database of a large commercial insurer. Patients with bipolar disorder were more likely to be high cost savers than individuals with other mood and anxiety disorders.

Funding Acknowledgements: Genomind

129

\section{Medication Assisted Treatment Protocol}

David Michael Mathis, DO FASAM DABAM

ABSTRACT: This article describes a protocol to be able to utilize medication assisted treatment options for patients dependent on opioids. The first step is using a 15-day Klonopin taper for effect detox of acute opioid withdrawal. Once detoxed, the patient can be started on low dose methadone or low dose Buprenorphine. Titration above $40 \mathrm{mg}$ of Methadone, or $8 \mathrm{mg}$ of buprenorphine will usually not be needed. Buprenorphine is utilized as the mono product, Subutex. Avoiding Suboxone eliminates the risk of reemergence of acute opioid withdrawal symptoms.

A description of how to transition to Naltrexone is provided. There are some differences between Methadone and Buprenorphine in the transition to Naltrexone. Once the patient is transitioned to Naltrexone, the stage is set for the patient to be able to get off medication assisted treatment.

\section{0}

Temporal Lobe Epilepsy: Is It in Your Differential Diagnosis? Two Case Reports

Debra $K$ Avery, PMHNP-BC, $B S A^{\prime}$; and Julie Brann D. Min., PMHNP-BC'

${ }^{1}$ Independent Practitioner, Colorado Springs, CO

ABSTRACT: Temporal Lobe Epilepsy: Is it in your differential diagnosis? Two Case Reports

OBJECTIVE: Temporal Lobe Epilepsy (TLE), also referred as Complex Partial Seizures, is a medical diagnosis that must be considered in the pediatric, adolescent, and adult population presenting for psychiatric care. Mood disorders are common in people with epilepsy, with a prevalence rate of 20 to $50 \%$. Scant literature exists that seeks specifically to enhance our knowledge of the similarities and subtle differences between TLE, Bipolar Disorder (BD) and Post Traumatic Stress Disorder (PTSD). Our objective is to describe an adolescent and adult case; one initially diagnosed as BD, the other PTSD, when in fact, it was TLE. We aim to illustrate that misdiagnosis and failure to diagnose are common. The provider must engage in a thorough review of systems and consider
TLE in the differential diagnosis. A delay in the appropriate diagnosis and treatment can lead to a substantial amount of adverse effects and worsening of symptoms and negatively impact one's quality of life.

METHOD: Two case studies; an adolescent and one adult, diagnosed with BD and the other PTSD. Both endorsed a history of symptoms indicative of TLE. Key assessment findings and screening diagnostics alerted us to the differential diagnosis of TLE. The overlap of the symptom presentation is described.

RESULTS: TLE and many psychiatric conditions often present with overlapping symptoms. Patients have the potential to present with absence seizures, unprovoked irritability, oppositionality, aggression, anger, paroxysmal anxiety, somatic symptoms such as headaches, nausea, burning in the abdomen, stereotyped movements or behaviors, hypergraphia bizarre or incongruous affect, symptoms of fear, disturbed sleep, tearfulness, memory problems, déjà vu, fugue states, changes in cognition, inability to concentrate, fatigue, auditory and visual hallucination and bad temper. Our differential diagnosis of TLE was confirmed with electroencephalogram (EEG). By prescribing the appropriate medications to these two individuals, they were able to experience improved moods, become more productive in society, working, attending church, family outings, etc. They were weaned off their antipsychotic medications, of which an abundance of troubling side effects is now a non-issue.

CONCLUSIONS: A delay in the proper diagnosis of TLE can have a significant negative impact on the adolescent and adult population. A need exists to educate mental health professionals on the overlap of symptoms of TLE and psychiatric disorders. The significant issue at hand is that they may not be receiving adequate or appropriate medications. Considering TLE in the differential diagnosis of presenting mood instability ensures our patients they are getting the basics of psychiatric care; which always emphasizes ruling out medical conditions first.

131

\section{A Marionettist Pulling My Strings: A Case of Buprenorphine-induced Chorea}

\author{
Dev Patel ${ }^{\prime, 2}$; Ishandeep Gandhi'; Faisal Malek'; \\ Camille Olechowski $i^{3}$ and Alan R. Hirsch ${ }^{4}$ \\ ${ }^{1}$ Aureus University School of Medicine, Oranjestad, Aruba \\ ${ }^{2}$ Illinois Center for Neurological and Behavioral \\ Medicine, Des Plaines, Illinois \\ ${ }^{3}$ Trinity School of Medicine, St. Vincent and The \\ Grenadines \\ ${ }^{4}$ Illinois Center for Neurological and Behavioral \\ Medicine, Des Plaines, Illinois.
}


ABSTRACT: Introduction: Choreaform movements provoked by opiates is an infrequent adverse event. Buprenorphine induction of chorea has not heretofore been described. Such a case is presented.

METHOD: Case Study: A 38-year-old female presented with a decade long history of alcohol, cocaine, benzodiazepine, and heroin abuse. The patient was insufflating 1.5 grams of heroin daily. On presentation, she was actively withdrawing, scoring 17 on the Clinical Opioid Withdrawal Scale. Urine toxicology screening was positive for opiates, cocaine, and cannabinoids. Buprenorphine $4 \mathrm{mg}$ sublingual was initiated. Within one hour, she observed, "My legs were moving uncontrollably as if I was a marionette." These dance-like movements were isolated to both legs and gradually resolved after discontinuation of buprenorphine: most of the movements manifested in the first 8 hours, and dissipated over the next 2 days. She did have similar movements after treatment with quetiapine during a previous hospitalization, years earlier.

RESULTS: Abnormalities in physical examination: General: goiter, bilateral palmar erythema. Neurological examination: Cranial Nerve (CN) Examination: CN I: Alcohol Sniff Test: 2 (anosmia). Motor Examination: Drift testing: mild right pronator drift. Reflexes: 3+ bilateral lower extremities. Neuropsychiatric Examination: Clock Drawing Test: 3 (abnormal). Animal Fluency Test: 18 (normal). Go-No-Go Test 6/6 (normal).

DISCUSSION: Buprenorphine induced chorea could be a result of partial mu-opioid agonism, or kappa and delta receptor antagonism (Burke, 2018; Cowan, 1977). $\mathrm{Mu}-$ opioid receptor activation causes increased dopamine turnover in the nigrostriatum, which is responsible for locomotor sensitization (Campos-Jurado, 2017). With the addition of mu-opioid receptor modulation of dopamine release, kappa-opioid receptor alters various neurotransmitters in the basal ganglia, potentiating hyperkinetic movements. Buprenorphine's choreiformogenic action may be due to kappaopioid receptors ability to augment neurotransmission in the striatum (Escobar, 2017; Bonnet, 1998). The combination of simultaneous activity of these three opioid receptors may cause chorea, since they act to modulate dopamine, glutamate, and GABA in the direct and indirect pathways within the basal ganglia (Abin, 1989; Cui, 2013; Allouche, 2014; Trifilieff, 2013). This patient's history of heroin and cocaine use may have caused supersensitization of dopamine receptors (Memo, 1981), provoking hyperkinesia. Involvement of substance-induced sensitization with concurrent kappa-opioid receptor neurotransmitter augmentation in direct and indirect pathways in the basal ganglia may have primed our patient to the development of chorea after buprenorphine administration. Further investigation for the presence of extrapyramidal movements in those undergoing buprenorphine treatment is warranted.

132

\section{Treating Comorbid Childhood Bipolar Disorder and ADHD}

Darlyne G. Nemeth, Ph.D., M.P., M.P.A.P. ${ }^{\prime}$; and Kayla Mckenzie Chustz, B.S. ${ }^{2}$

${ }^{1}$ Clinical, Medical, and Neuropsychologist; The Neuropsychology Center of Louisiana; Baton Rouge, LA ${ }^{2}$ Clinical Assistant; The Neuropsychology Center of Louisiana; Baton Rouge, LA

ABSTRACT: Objectives: According to Nemeth et al. (2011), pediatric mania is difficult to distinguish from childhood hyperactivity. Both share 3 common symptoms: distractibility, motoric hyperactivity, and talkativeness (Biederman, 2000). Oftentimes, children are referred from their pediatrician due to a lack of appropriate response to stimulant medication. Pediatricians have learned that merely raising the dose or changing the stimulant does not work. A thorough neuropsychological evaluation often reveals Bipolar Mania. They may have comorbid Bipolar Disorder and ADHD. This poster paper will examine measures that can assist in this important differential diagnosis as well as offer treatment options, including medication management.

METHODS: This case study includes three pediatric patients diagnosed with Childhood Bipolar Disorder and ADHD. A comprehensive psychoeducational assessment was conducted for each of the patients, which resulted in this comorbid diagnosis.

RESULTS: One of the most helpful measures was the TOVA. When a child's attention and impulsivity scores are normal, and response time and variability scores are abnormal, both on and off medication, that is an indication of a mood disorder (Nemeth et al., 2007). These children also performed poorly on measures of processing speed, and verbal learning and interference tasks (Henin et al., 2007). Measures of affect and personality were important diagnostically. A combination of Amantadine and either Clonidine HCL ER or Propranolol, as prescribed by a medical psychologist, were found to be effective in controlling the symptoms of this comorbid diagnosis.

CONCLUSIONS: An evaluation of children's intellectual, attentional, behavioral, mood, and personality functioning is crucial for a differential diagnosis. In cases of 